of meters of ice of the Greenland or Antarctic ice sheets, whose mean surface temperatures are necessarily the mean annual ambient atmospheric temperature; except that interior heat of the earth, flowing up into the glacier from below, cannot flow up through to the surface of a thick glacier fast enough to be completely carried off, and some of it is then trapped in the lower levels of such polar glaciers, eventually warming their lower levels up to pressure-melting-point temperature.

Hence it follows that as all glaciers, both temperate and polar, are at least in part at pressuremelting-point, all glaciers, both temperate and polar, do possess flowability which is a function of pressure-and hence, are capable of extrusion flow.

It is true that copious melt water or solar heat on the surface of certain temperate glaciers may so accelerate the melting of the impure ice films between crystals close to the surface, as to permit the surface portion of such "over-wet" glaciers to flow actually faster than their lower parts. This does not deny the validity of extrusion flow; it means only that the surface portion may occasionally possess a condition enabling it, temporarily and locally, to outstrip the extrusion flow of its under part. But meanwhile, everywhere, in Alpine and Himalayan valley glaciers or névés - or in Greenland or Antarctic ice sheets, extrusion flow is the mechanism by which the beautiful principles of pressure-melting-point, aided by the result of concentration of solutes during crystal growth, together permit glacier motion as it operates to-day, as it operated in Pleistocene, in Triassic, and in earlier ages, beneath a (sometimes) thin covering of more friable, frozen surface ice.

NotE.-The unexplained further finding of Renaud (supra) that the amount of solute per cm. ${ }^{3}$ of ice, decreases steadily from firn to snout, bears testimony to the above process that decrease in salts in the outer edge of ice crystals, progressive from firn to snout, is due to their being leached out, as those thin films of "saline" ice are the first to be forced to melt, exactly as would be expected, from the above mechanism.

\title{
REPLY TO MR. JOEL E. FISHER'S COMMENTS
}

\author{
By J. F. NYE \\ (Cavendish Laboratory, Cambridge)
}

Mr. Fisher's remarks raise two questions :

(I) Does the shear stress necessary to cause a given rate of strain in ice decrease with increasing hydrostatic pressure?

(2) If the answer to (I) is "Yes," could this cause extrusion flow?

I do not think it is possible to come to a definite conclusion on question (I) without more detailed reasoning than $\mathrm{Mr}$. Fisher has given. In order to prove that pressure melting can take place in a glacier it is surely essential to show that enough heat to provide the latent heat of melting can flow in within the time available. This would be decided by many factors, among them the rate of flow of heat from the earth, the mean temperature gradient in the glacier and the length of time it takes a given ice crystal to travel from the surface down to its maximum depth (a period of increasing pressure) and up again (a period of decreasing pressure). In the absence of a detailed examination of these points my own opinion was that extensive pressure melting would be unlikely (I would not be so rash as to say it is impossible) and that was why I thought it right to assume in my paper that deformation was independent of pressure. I did not, as Mr. Fisher says, conclude this "by direct mathematics." I assumed it for the purpose of mathematical calculation.

In answer to the second question, I think that even if ice does deform more easily under pressure it would still not show extrusion flow in the simple cases I discussed. I explained this, perhaps in too condensed a way, on page 565 of my paper. I rejected extrusion flow in these simple cases, not because that theory requires ice to deform more easily at depth but because it is based on unsound mechanics. Let me try to make this clear by an example. 
Fig. I (below) shows the profile of an ideal ice cap on a horizontal bed, say with the dimensions of the east-west profile of Greenland. Let us suppose that extrusion flow is occurring. I take this to mean that if a line $A B$ is drawn at a suitable depth the ice just below $A B$ is moving outwards faster than the ice just above $\mathrm{AB}$. It follows that the ice below $\mathrm{AB}$ is exerting an outward force on the ice above $A B$, as shown by the arrow. The only other force acting on the segment $A B C$ in the horizontal direction (assuming a two-dimensional problem) is the normal pressure acting on AC. (I think Mr. Fisher would agree that this last force is a pressure rather than a tension, for his thesis depends on the existence of such a pressure.) It will be seen that the horizontal forces on $\mathrm{ABC}$ are all in the outward direction. $\mathrm{ABC}$ will therefore acclerate to the right. We may estimate the magnitude of this acceleration as follows. Consider unit thickness of ice perpendicular to the diagram. Let $\mathrm{AC}=z$ and $\mathrm{AB}=l$. The normal pressure on $\mathrm{AC}$ increases from o at $\mathrm{C}$ to approximately $\rho g z$ at $A$; the average value is $\frac{1}{4} \rho g z$ and the total normal force on $A C$ is thus $\frac{1}{2} \rho g z^{2}$. The mass of $\mathrm{ABC}$ is certainly less than $\rho z l$. Therefore the force on $\mathrm{AC}$ alone, even without the aid of the force on $\mathrm{AB}$, produces an acceleration of the segment $\mathrm{ABC}$ of at least

$$
\frac{1}{2} \rho g z^{2} / \rho z l=\frac{g z}{2 l} \text {. }
$$

With $g=98 \mathrm{r} \mathrm{cm} . / \mathrm{sec}^{2}, 2 l=880 \mathrm{Km}$., and $z=1500 \mathrm{~m}$. (taking only half the maximum height of Greenland so as not to overestimate the acceleration) the acceleration is $1 \cdot 7 \mathrm{~cm} . / \mathrm{sec}^{2}$. This is a

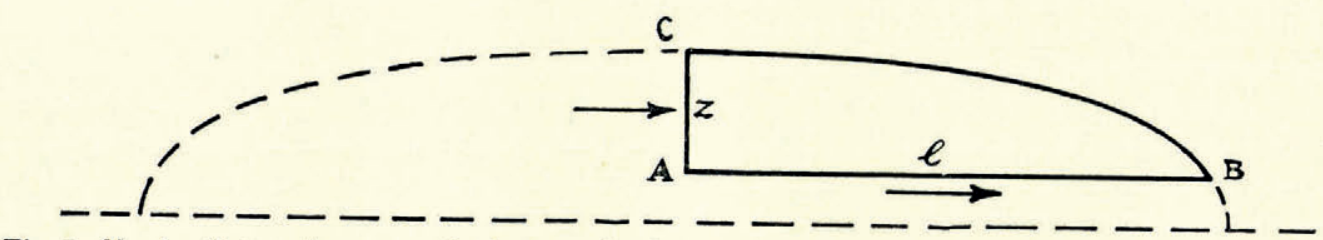

Fig. I. Showing that an ice cap moving by extrusion flow is not in statical equilibrium. Vertical scale exaggerated

very high figure. Starting from rest under this acceleration a particle of ice would move the distance $A B$ in 2 hours. Or, if the acceleration were sustained for 100 years, which is not long compared with the lifetime of a piece of ice in Greenland, the ice would reach nearly one-fifth the velocity of light. Such absurd results show, I think, that to explain the continuing existence of the ice cap it is absolutely necessary to have a force opposing the pressure on AC. This can only be done by reversing in direction the shear stress on AB. The motion is in fact quasi-static and one may say that the outward pressure on $\mathrm{AC}$ just balances the inward shear force on $\mathrm{AB}$. It then follows that, since direction of shear stress determines the direction of velocity gradient, the ice immediately above $\mathrm{AB}$ is moving outwards faster than the ice below. These are the reasons why I think that, even if hydrostatic pressure does make ice easier to deform, extrusion flow in an ideal ice cap of this sort is a mechanical absurdity.

For a valley glacier a rather similar statical argument may be used. It shows that the tendency of the lower layers to carry the upper layers forward with them can only be opposed either by a longitudinal tensile stress or by transverse shear stresses resulting from the drag of the upper parts of the valley sides. In actual glaciers these stresses would need to be very high, many times the tensile strength of ice. (In my paper extrusion flow did not appear even as a theoretical possibility because I explicitly neglected the drag of the valley sides.) I suppose it might be possible to achieve the extrusion result with a sufficiently deep and narrow valley of the right shape, but I feel that anyone who wishes to make out a case for a theory of extrusion flow must first show that the proposed system is in statical equilibrium, and then that the forces required for this equilibrium can in fact be sustained by the ice. 